CERN-TH/97-148

hep-ph/9707217

\title{
Theory of Beauty Lifetimes
}

\author{
M. Neubert \\ Theory Division, CERN, CH-1211 Geneva 23, Switzerland
}

\begin{abstract}
A critical review of the theoretical understanding of the lifetimes of beauty hadrons is given. A model-independent analysis using the heavy-quark expansion allows for a description of the lifetime ratios $\tau\left(B^{-}\right) / \tau\left(B^{0}\right)$ and $\tau\left(\Lambda_{b}\right) / \tau\left(B^{0}\right)$ in a small region of parameter space. It is demonstrated that the lifetime ratio $\tau\left(B^{-}\right) / \tau\left(B^{0}\right)$ cannot be used to extract the decay constant of the $B$ meson. Implications for the semileptonic branching ratio and charm yield in $B$ decays are pointed out.
\end{abstract}

Invited talk presented at the Second International Conference on $B$ Physics and CP Violation Honolulu, Hawaii, 24-27 March 1997

CERN-TH/97-148

July 1997 


\section{Introduction}

The theoretical description of inclusive decays of hadrons containing a heavy quark exploits two observations [1]-[6]: bound-state effects related to the initial state can be calculated using the heavy-quark expansion, and the fact that the final state consists of a sum over many hadronic channels eliminates the sensitivity to the properties of individual final-state hadrons. The second feature rests on the hypothesis of quark-hadron duality, i.e. the assumption that decay rates are calculable in QCD after a "smearing" procedure has been applied [7]. In semileptonic decays the integration over the lepton spectrum provides a smearing over the invariant hadronic mass of the final state (socalled global duality), whereas for nonleptonic decays, where the total hadronic mass is fixed, the summation over many hadronic final states provides an averaging (so-called local duality). The calculation of the lifetimes of heavy hadrons relies on the stronger assumption of local duality.

At present, quark-hadron duality cannot be derived from first principles, although some interesting attempts to address the problem have been discussed recently [8, 9]. The validity of global duality (at energies even lower than those relevant in $B$ decays) has been tested experimentally using high-precision data on hadronic $\tau$ decays [10]. The study of beauty lifetime ratios provides a test of local duality at energies of order a few $\mathrm{GeV}$. Unlike the semileptonic branching ratio of $B$ mesons, lifetime ratios are insensitive to possible new physics contributions and thus probe directly low-energy strong interactions. The common folklore is that the current experimental value $\tau\left(\Lambda_{b}\right) / \tau(B) \approx 0.8$ cannot be accommodated by theory, indicating sizeable duality violations. If this were to be true, we could not trust the predictions of the heavy-quark expansion for other inclusive quantities, such as the semileptonic branching ratio and charm yield in $B$ decays, which are considered sensitive probes of new physics. A calibration of the theoretical uncertainties in the predictions for these quantities is therefore most important.

Here we shall reconsider the theoretical description of beauty lifetime ratios taking a conservative point of view, which avoids making uncontrolled approximations [11]. We concentrate on the ratios $\tau\left(B^{-}\right) / \tau\left(B^{0}\right)$ and $\tau\left(\Lambda_{b}\right) / \tau\left(B^{0}\right)$, for which precise experimental data exist. Our model-independent approach is less ambitious than previous analyses in that it gives up some predictive power. Yet, we find it important to question commonly made assumptions before concluding a failure of the heavy-quark expansion. We find that at present it is still possible to reproduce the experimental data on beauty lifetimes in the framework of the heavy-quark expansion without invoking duality violations. Only a better control of theoretical uncertainties could help to clarify the situation.

\section{Heavy-Quark Expansion}

The inclusive decay width of a hadron $H_{b}$ containing a $b$ quark can be written in the form

$$
\Gamma\left(H_{b} \rightarrow X\right)=\frac{1}{M_{H_{b}}} \operatorname{Im}\left\langle H_{b}|\mathbf{T}| H_{b}\right\rangle
$$


where the transition operator $\mathbf{T}$ is given by

$$
\mathbf{T}=i \int \mathrm{d}^{4} x T\left\{\mathcal{L}_{\text {eff }}(x), \mathcal{L}_{\text {eff }}(0)\right\} .
$$

Here $\mathcal{L}_{\text {eff }}$ is the effective weak Lagrangian renormalized at the scale $m_{b}$ [12, 13]. The leading contributions to the transition operator are given by the two-loop diagrams shown on the left-hand side in Fig. 1. Because of the large mass of the $b$ quark, the momenta flowing through the internal propagator lines are large. It is thus possible to construct an operator product expansion (OPE) for the transition operator, in which it is represented as a series of local operators containing the $b$-quark fields. The operator with the lowest dimension is $\bar{b} b$. It arises by contracting the internal lines of the first diagram. The only gauge-invariant operator with dimension 4 is $\bar{b} i \not D b$; however, the equations of motion imply that this operator can be replaced by $m_{b} \bar{b} b$. The first operator that is different from $\bar{b} b$ has dimension 5 and contains the gluon field. It arises from diagrams in which a gluon is emitted from one of the internal lines, such as the second diagram shown in Fig. 1. From dimension 6 on, a large number of operators appears. For dimensional reasons, the matrix elements of higher-dimensional operators are suppressed by inverse powers of the $b$-quark mass. Thus, the total inclusive decay rate (i.e. the inverse lifetime) of a hadron $H_{b}$ can be written as [2, 3]

$$
\Gamma\left(H_{b}\right)=\frac{G_{F}^{2} m_{b}^{5}\left|V_{c b}\right|^{2}}{192 \pi^{3}}\left\{c_{3}\langle\bar{b} b\rangle+c_{5} \frac{\left\langle\bar{b} g_{s} \sigma_{\mu \nu} G^{\mu \nu} b\right\rangle}{m_{b}^{2}}+\sum_{n} c_{6}^{(n)} \frac{\left\langle O_{6}^{(n)}\right\rangle}{m_{b}^{3}}+\ldots\right\}
$$

where the prefactor arises from the loop integrations, $c_{i}$ are calculable coefficient functions, and $\langle O\rangle$ are the (normalized) forward matrix elements between $H_{b}$ states. These matrix elements can be systematically expanded in powers of $1 / m_{b}$ using the heavy-quark effective theory (HQET) [14]. The result is [2, 3]

$$
\begin{aligned}
\langle\bar{b} b\rangle & =1-\frac{\mu_{\pi}^{2}\left(H_{b}\right)-\mu_{G}^{2}\left(H_{b}\right)}{2 m_{b}^{2}}+O\left(1 / m_{b}^{3}\right), \\
\left\langle\bar{b} g_{s} \sigma_{\mu \nu} G^{\mu \nu} b\right\rangle & =2 \mu_{G}^{2}\left(H_{b}\right)+O\left(1 / m_{b}\right),
\end{aligned}
$$

where $\mu_{\pi}^{2}\left(H_{b}\right)$ and $\mu_{G}^{2}\left(H_{b}\right)$ are the matrix elements of the heavy-quark kinetic energy and chromomagnetic interaction inside the hadron $H_{b}$, respectively [15]. Inserting these relations into (3) and taking the ratio of two lifetimes yields [11

$$
\begin{aligned}
\frac{\tau\left(B^{-}\right)}{\tau\left(B^{0}\right)} & =1+O\left(1 / m_{b}^{3}\right) \\
\frac{\tau\left(\Lambda_{b}\right)}{\tau\left(B^{0}\right)} & =1+\frac{\mu_{\pi}^{2}\left(\Lambda_{b}\right)-\mu_{\pi}^{2}(B)}{2 m_{b}^{2}}+c_{G} \frac{\mu_{G}^{2}\left(\Lambda_{b}\right)-\mu_{G}^{2}(B)}{m_{b}^{2}}+O\left(1 / m_{b}^{3}\right) \\
& =(0.98 \pm 0.01)+O\left(1 / m_{b}^{3}\right),
\end{aligned}
$$

where $c_{G}=-\frac{1}{2}-2 c_{5} / c_{3} \approx 1.2$, and isospin symmetry is assumed for the matrix elements of $B^{-}$and $B^{0}$ mesons. The hadronic parameters appearing at order $1 / m_{b}^{2}$ in the 

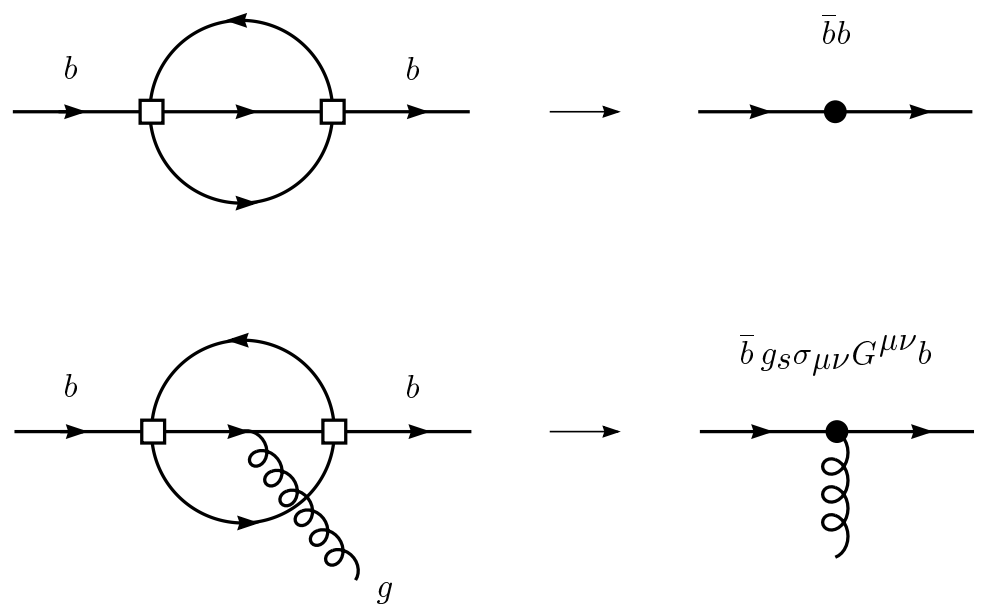

Figure 1: Leading contributions to the transition operator $\mathbf{T}$ (left), and the corresponding operators in the OPE (right). The open squares represent a four-fermion interaction of the effective weak Lagrangian.

ratio $\tau\left(\Lambda_{b}\right) / \tau\left(B^{0}\right)$ can all be determined from spectroscopy. The matrix elements of the chromomagnetic interaction are given by

$$
\mu_{G}^{2}(B)=\frac{3}{4}\left(M_{B^{*}}^{2}-M_{B}^{2}\right) \approx 0.36 \mathrm{GeV}^{2}, \quad \mu_{G}^{2}\left(\Lambda_{b}\right)=0
$$

where the second relation follows from the fact that in the ground-state $\Lambda_{b}$ baryon the light constituents have total spin zero. The individual matrix elements of the kinetic energy are not physical quantities because of renormalon ambiguities [16]; however, their difference obeys the relation

$$
\mu_{\pi}^{2}\left(\Lambda_{b}\right)-\mu_{\pi}^{2}(B)=-\frac{M_{B} M_{D}}{2}\left(\frac{M_{\Lambda_{b}}-M_{\Lambda_{c}}}{M_{B}-M_{D}}-\frac{3}{4} \frac{M_{B^{*}}-M_{D^{*}}}{M_{B}-M_{D}}-\frac{1}{4}\right)
$$

which implies $\mu_{\pi}^{2}\left(\Lambda_{b}\right)=\mu_{\pi}^{2}(B)$ up to small corrections of order $0.01 \mathrm{GeV}^{2}$.

The model-independent theoretical predictions in (5) may be compared with the average experimental values for the lifetime ratios, which are [17]

$$
\frac{\tau\left(B^{-}\right)}{\tau\left(B^{0}\right)}=1.06 \pm 0.04, \quad \frac{\tau\left(\Lambda_{b}\right)}{\tau\left(B^{0}\right)}=0.79 \pm 0.05 .
$$

Whereas the first ratio is in good agreement with the theoretical expectation, the low value of the lifetime of the $\Lambda_{b}$ baryon constitutes a puzzle. To understand the fine structure of the lifetime ratios requires to go further in the $1 / m_{b}$ expansion. At order $1 / m_{b}^{3}$ in the expansion of nonleptonic decay rates there appear four-quark operators whose matrix elements explicitly depend on the flavour of the spectator quark(s) in the hadron $H_{b}$, and hence differentiate between hadrons with different light constituents. These nonspectator effects receive a phase-space enhancement factor of $O\left(16 \pi^{2}\right)$ with respect 
to the leading terms in the OPE, which makes them more important than the corrections of order $1 / m_{b}^{2}$. This can be seen from Fig. 目, which shows that the corresponding contributions to the transition operator arise from one-loop rather than two-loop diagrams. In total, a set of four four-quark operators is induced by nonspectator effects. They are

$$
\begin{aligned}
O_{1} & =\bar{b} \gamma_{\mu}\left(1-\gamma_{5}\right) q \bar{q} \gamma^{\mu}\left(1-\gamma_{5}\right) b \\
O_{2} & =\bar{b}\left(1-\gamma_{5}\right) q \bar{q}\left(1+\gamma_{5}\right) b \\
T_{1} & =\bar{b} \gamma_{\mu}\left(1-\gamma_{5}\right) t_{a} q \bar{q} \gamma^{\mu}\left(1-\gamma_{5}\right) t_{a} b \\
T_{2} & =\bar{b}\left(1-\gamma_{5}\right) t_{a} q \bar{q}\left(1+\gamma_{5}\right) t_{a} b
\end{aligned}
$$

where $q$ is a light quark, and $t_{a}$ are the generators of colour $\mathrm{SU}(3)$. Since hadronic matrix elements of four-quark operators are notoriously difficult to calculate, one needs to parametrize them. For the $B$-meson matrix elements renormalized at the scale $m_{b}$, we define [11

$$
\left\langle B\left|O_{i}\right| B\right\rangle_{\mu=m_{b}} \equiv B_{i} f_{B}^{2} M_{B}^{2}, \quad\left\langle B\left|T_{i}\right| B\right\rangle_{\mu=m_{b}} \equiv \varepsilon_{i} f_{B}^{2} M_{B}^{2},
$$

where $f_{B}$ is the decay constant of the $B$ meson. The values of the dimensionless hadronic parameters $B_{i}$ and $\varepsilon_{i}$ are currently not known. Ultimately, they may be calculated using some field-theoretic approach such as lattice gauge theory. Some (but not all) of these parameters may also be extracted from a precise measurement of the lepton spectrum in the endpoint region of semileptonic $B$ decays [18]. We note that in the vacuum saturation (or factorization) approximation [19] $B_{i}=1$ and $\varepsilon_{i}=0$ at some scale $\mu$, where the approximation is assumed to hold. More generally, the large- $N_{c}$ counting rules of QCD imply

$$
B_{i}=O(1), \quad \varepsilon_{i}=O\left(1 / N_{c}\right) .
$$

The theoretical expressions can also be written in terms of parameters $B_{i}(\mu)$ and $\varepsilon_{i}(\mu)$ renormalized at a scale $\mu \neq m_{b}$, using evolution equations for the hadronic matrix elements in the HQET. Such a rewriting does not induce any uncertainty, as it is simply a reparametrization of the results. Still, it may be appropriate to use a lower renormalization point if model calculations of the parameters $B_{i}$ and $\varepsilon_{i}$ are used. To give an example, we relate the parameters in (10) to ones renormalized at a low hadronic scale $\mu_{\text {had }}$ chosen such that $\alpha_{s}\left(\mu_{\text {had }}\right)=0.5$. In defining these parameters, we also renormalize the decay constant $f_{B}$ at the scale $\mu_{\text {had }}$. Then the relations are [11]

$$
\begin{aligned}
& B_{i} \approx 1.01 B_{i}\left(\mu_{\mathrm{had}}\right)-0.24 \varepsilon_{i}\left(\mu_{\mathrm{had}}\right) \\
& \varepsilon_{i} \approx-0.05 B_{i}\left(\mu_{\mathrm{had}}\right)+0.73 \varepsilon_{i}\left(\mu_{\mathrm{had}}\right) .
\end{aligned}
$$

Next consider the matrix elements of the four-quark operators between $\Lambda_{b}$ baryons. Heavy-quark spin symmetry implies the relations $\left\langle O_{2}\right\rangle=-\frac{1}{2}\left\langle O_{1}\right\rangle$ and $\left\langle T_{2}\right\rangle=-\frac{1}{2}\left\langle T_{1}\right\rangle$ [11], which reflect the fact that the light degrees of freedom in a $\Lambda_{b}$ baryon are in a state with total spin zero. This leaves two independent matrix elements of the operators $O_{1}$ and 


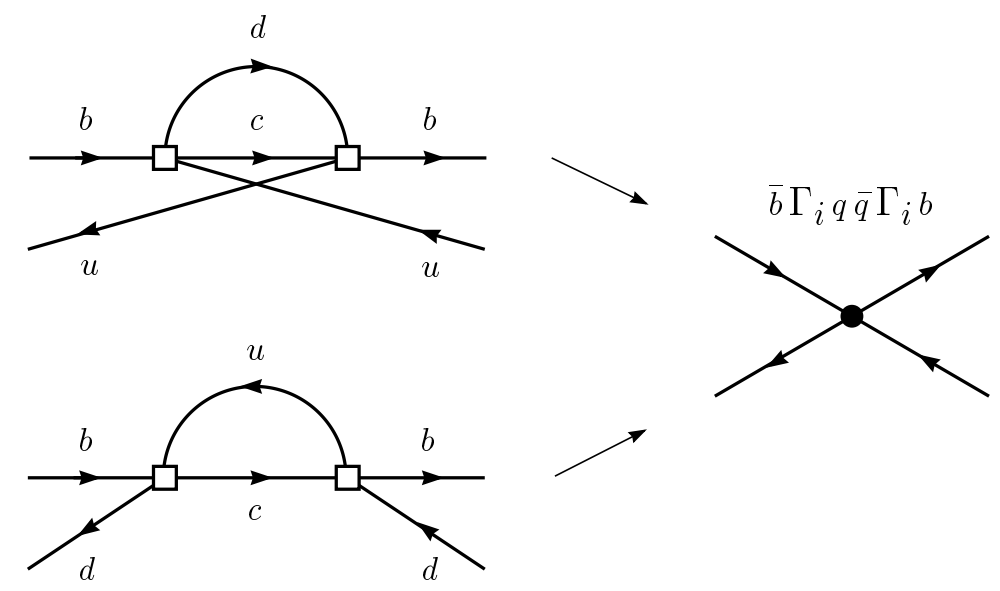

Figure 2: Nonspectator contributions to the transition operator $\mathbf{T}$ (left), and the corresponding operators in the OPE (right). $\Gamma_{i}$ denote combinations of Dirac and colour matrices.

$T_{1}$. It is convenient to introduce the operator $\widetilde{O}_{1}=\bar{b}^{i} \gamma_{\mu}\left(1-\gamma_{5}\right) q^{j} \bar{q}^{j} \gamma^{\mu}\left(1-\gamma_{5}\right) b^{i}$ instead of $T_{1}\left(i, j\right.$ are colour indices) by making use of the Fierz identity $T_{1}=-\frac{1}{6} O_{1}+\frac{1}{2} \widetilde{O}_{1}$. The analogue of the vacuum saturation approximation in the case of baryons is the valence-quark assumption, in which the colour of the quark fields in the operators is identified with the colour of the quarks inside the baryon. Since the colour wave function of a baryon is totally antisymmetric, the matrix elements of $O_{1}$ and $\widetilde{O}_{1}$ differ in this approximation only by a sign. Hence, it is natural to define

$$
\left\langle\Lambda_{b}\left|\widetilde{O}_{1}\right| \Lambda_{b}\right\rangle_{\mu=m_{b}} \equiv-\widetilde{B}\left\langle\Lambda_{b}\left|O_{1}\right| \Lambda_{b}\right\rangle_{\mu=m_{b}},
$$

with $\widetilde{B}=1$ in the valence-quark approximation. For the baryon matrix element of $O_{1}$ itself, we adopt a parametrization guided by the quark model and write

$$
\frac{1}{2 M_{\Lambda_{b}}}\left\langle\Lambda_{b}\left|O_{1}\right| \Lambda_{b}\right\rangle_{\mu=m_{b}} \equiv-\frac{f_{B}^{2} M_{B}}{48} r .
$$

In the quark model $r$ is the ratio of the squares of the wave functions determining the probability to find a light quark at the location of the $b$ quark inside the $\Lambda_{b}$ baryon and the $B$ meson [20, 21]. The parameter $r$ is by far the most uncertain one entering the theoretical formulae for beauty lifetimes. Theoretical estimates range from $r \approx 0.1-$ 0.5 [22, 23] to $r \approx 1-2$ [24]. In the quark model, $r$ can be related to the ratio of the spin splittings between $\Sigma_{b}$ and $\Sigma_{b}^{*}$ baryons and $B$ and $B^{*}$ mesons, leading to [24]

$$
r \approx \frac{4}{3} \frac{M_{\Sigma_{b}^{*}}^{2}-M_{\Sigma_{b}}^{2}}{M_{B^{*}}^{2}-M_{B}^{2}} .
$$

Using the (still preliminary) result $M_{\Sigma_{b}^{*}}-M_{\Sigma_{b}}=(56 \pm 16) \mathrm{MeV}$ reported by the DELPHI Collaboration [25], one then obtains $r \approx 1.8 \pm 0.5$. 


\section{Model-Independent Analysis of Lifetime Ratios}

Including the nonspectator contributions of order $1 / m_{b}^{3}$ allows us to refine the results in (5) at the prize of introducing several unknown hadronic parameters. We obtain [1]

$$
\begin{aligned}
\frac{\tau\left(B^{-}\right)}{\tau\left(B^{0}\right)} & =1+\xi\left[k_{1} B_{1}+k_{2} B_{2}+k_{3} \varepsilon_{1}+k_{4} \varepsilon_{2}\right] \\
\frac{\tau\left(\Lambda_{b}\right)}{\tau\left(B^{0}\right)} & =0.98+\xi\left[p_{1} B_{1}+p_{2} B_{2}+p_{3} \varepsilon_{1}+p_{4} \varepsilon_{2}+\left(p_{5}+p_{6} \widetilde{B}\right) r\right]
\end{aligned}
$$

where $\xi=\left(f_{B} / 200 \mathrm{MeV}\right)^{2}$. The coefficients $k_{i}$ and $p_{i}$ depend on the quark-mass ratio $m_{c} / m_{b}$ and the values of the Wilson coefficients $c_{ \pm}\left(m_{b}\right)$ appearing in the effective weak Lagrangian. They also depend on the perturbative scheme adopted in the calculation. So far, we have discussed the scheme in which the standard QCD evolution is used to obtain the effective weak Lagrangian at the scale $m_{b}$, where the matrix elements in the heavy-quark expansion are evaluated. To investigate the perturbative uncertainties, one may also renormalize the weak Lagrangian at a scale $\mu=\kappa m_{b}$ with $\kappa=O(1)$, then perform the heavy-quark expansion, and finally evolve the hadronic matrix elements from $\kappa m_{b}$ to $m_{b}$ using evolution equations in the HQET. If it was not for the truncation of perturbation theory, the two procedures would yield to the same result. The dependence of $k_{i}$ and $p_{i}$ on the choice of $\kappa$ is therefore a measure of the truncation error. In Table 1, we give the values of these coefficients for the three choices $\kappa=1 / 2,1$ and 2 . Because of an accidental cancellation between the contribution from different operators in the effective weak Lagrangian, the coefficients $k_{1}$ and $p_{5}$ have a significant dependence on the choice of the "matching" scale, whereas the other coefficients are either negligibly small or stable with respect to variations of $\kappa$.

Table 1: Coefficients $k_{i}$ and $p_{i}$ appearing in the predictions for the lifetime ratios. We use $c_{+}\left(m_{b}\right)=0.86, c_{-}\left(m_{b}\right)=1.35$, and $m_{c} / m_{b}=0.29$

\begin{tabular}{|c|cccccc|}
\hline \hline$\kappa$ & $k_{1}$ & $k_{2}$ & $k_{3}$ & $k_{4}$ & & \\
\hline $1 / 2$ & +0.046 & 0.003 & -0.737 & 0.201 & & \\
1 & +0.021 & 0.004 & -0.699 & 0.195 & & \\
2 & -0.007 & 0.007 & -0.666 & 0.189 & & \\
\hline & $p_{1}$ & $p_{2}$ & $p_{3}$ & $p_{4}$ & $p_{5}$ & $p_{6}$ \\
\hline $1 / 2$ & -0.003 & 0.003 & -0.178 & 0.201 & -0.017 & -0.023 \\
1 & -0.003 & 0.004 & -0.173 & 0.195 & -0.012 & -0.021 \\
2 & -0.006 & 0.007 & -0.168 & 0.189 & -0.008 & -0.020 \\
\hline \hline
\end{tabular}

Previous analyses of beauty lifetimes [26, 27] have avoided the proliferation of unknown hadronic parameters by making strong model assumptions. The meson matrix elements of four-quark operators have been estimated using the vacuum saturation approximation at a low hadronic scale $\mu_{\text {had }}$ which, according to (12), yields $B_{i} \approx 1.0$ and 
$\varepsilon_{i} \approx-0.05$ at the scale $m_{b}$. The baryon matrix elements have been estimated adopting the valence-quark approximation $\widetilde{B}=1$ and assuming $r=O(1)$. These assumptions lead to the model predictions

$$
\frac{\tau\left(B^{-}\right)}{\tau\left(B^{0}\right)}=1+(0.05 \pm 0.03) \times\left(\frac{f_{B}}{200 \mathrm{MeV}}\right)^{2}, \quad \frac{\tau\left(\Lambda_{b}\right)}{\tau\left(B^{0}\right)} \approx 0.95 .
$$

Whereas the first relation is in good agreement with the experimental result in (8), the predicted value for the ratio $\tau\left(\Lambda_{b}\right) / \tau\left(B^{0}\right)$ is much larger than the experimental one. This discrepancy is referred to as the " $\Lambda_{b}$ lifetime puzzle".

It has been advocated in Refs. [27] that a precise measurement of the lifetime ratio $\tau\left(B^{-}\right) / \tau\left(B^{0}\right)$ would allow for a determination of the $B$-meson decay constant $f_{B}$ - a proposal which has been taken seriously by the DELPHI Collaboration [28]. Note that the ambiguity in the choice of the matching scale alone implies a large uncertainty in the coefficient in front of $f_{B}^{2}$ in (17), which is ignored in Refs. [27]. In addition, the numerical results for the coefficients $k_{i}$ in Table 1 show that assuming exact vacuum saturation is not justifiable in the case of the ratio $\tau\left(B^{-}\right) / \tau\left(B^{0}\right)$. To neglect in (16) the deviation $\Delta \varepsilon_{1}$ of the unknown parameter $\varepsilon_{1}$ from the value -0.05 predicted by factorization at a low scale would only be justified if $\left|\Delta \varepsilon_{1}\right| \ll 0.1$. The large- $N_{c}$ counting rules of QCD do not allow us to impose such a strong restriction.

Our goal here is to investigate in a model-independent way how the "predictions" in (17) are altered if model assumptions are avoided. To this end, we allow all hadronic parameters to vary within reasonable limits and use the large- $N_{c}$ counting rules and other theoretical prejudices only to restrict the allowed parameter ranges. To be specific, we take random distributions inside the following ranges: $B_{i}, \widetilde{B} \in[2 / 3,4 / 3]$, $\varepsilon_{i} \in[-1 / 3,1 / 3], r \in[0.25,2.5]$, and $\xi \in[0.8,1.2]$. For each parameter set, the lifetime ratios $\tau\left(B^{-}\right) / \tau\left(B^{0}\right)$ and $\tau\left(\Lambda_{b}\right) / \tau\left(B^{0}\right)$ are computed for the two perturbative schemes with $\kappa=1 / 2$ and 1 . The results are shown in Fig. 3 . In general, the scheme with $\kappa=1 / 2$ gives results closer to the experimental data. There are two important observations: first, only a small tail of the theoretical distribution for the ratio $\tau\left(\Lambda_{b}\right) / \tau\left(B^{0}\right)$ reaches into the region preferred by the experimental data; secondly, the predictions for the ratio $\tau\left(B^{-}\right) / \tau\left(B^{0}\right)$ have an almost flat distribution between 0.85 and 1.25 , indicating that without a better knowledge of the hadronic parameters it is not possible to predict this ratio with good precision. At present, not even the sign of the deviation from unity can be predicted without model assumptions, in stark contrast with the model-dependent result in (17).

In the next step, we focus on the parameter sets which give results within the $2 \sigma$ ellipse around the central experimental values. For these, the projected distributions for all hadronic parameters are shown in Fig. 1 . The distributions of $B_{1}$ and $B_{2}$ and, to a lesser extend, $\widetilde{B}$ and $\xi$ are rather flat, indicating that these parameters are little restricted by the data. The remaining three parameters are highly constrained, however. The parameter $\varepsilon_{1}$, in particular, is determined by the experimental data within a rather limited range: $\varepsilon_{1} \approx-(0.1 \pm 0.1)$, in good agreement with the expectation $\varepsilon_{1} \approx-0.05$ based on the vacuum saturation approximation. The distributions of the parameters $\varepsilon_{2}$ 

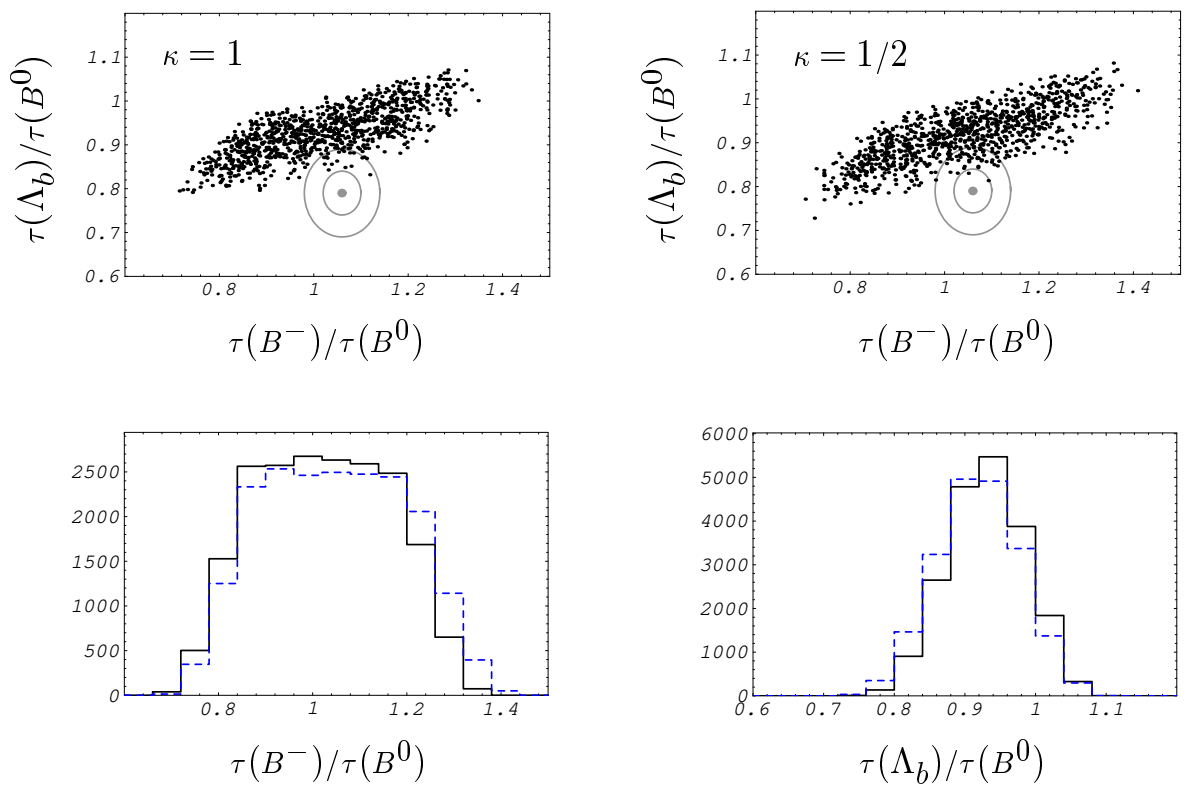

Figure 3: Theoretical results for the lifetime ratios for random choices of the hadronic parameters within the limits specified in the text. The ellipses in the upper plots show the $1 \sigma$ and $2 \sigma$ contours around the central experimental values. In the lower plots, the projections on the individual lifetime ratios are shown. Full lines correspond to $\kappa=1$, dashed ones to $\kappa=1 / 2$.

and $r$ are strongly correlated. To reproduce the experimental data requires to have $\varepsilon_{2}$ negative and $r \gtrsim 1$. The correlation between the two parameters in shown in the last plot (for the scheme with $\kappa=1 / 2$ ). If we assume that $\left|\varepsilon_{2}\right|<0.15$, for instance, then $r$ must be larger than about 1.25, which is higher than most expectations [22, 23]. To summarize, although some large hadronic parameters are needed to explain the observed pattern of beauty lifetimes, a conventional explanation of the $\Lambda_{b}$ lifetime puzzle cannot be excluded before reliable field-theoretic calculations of the matrix elements of four-quark operators become available.

\section{Conclusions and Implications}

The heavy-quark expansion, supplemented by the assumption of quark-hadron duality, provides the theoretical framework for a systematic calculation of the lifetimes and inclusive decay rates of hadrons containing a heavy quark. Whereas this formalism works well for the description of inclusive semileptonic decays, two potential problems related to nonleptonic decays have become apparent in recent years: the low experimental value of the lifetime ratio $\tau\left(\Lambda_{b}\right) / \tau\left(B^{0}\right)$, and the low values of the semileptonic branching ratio and charm yield in $B$ decays.

In order to obtain a detailed understanding of beauty lifetimes it is necessary to go 

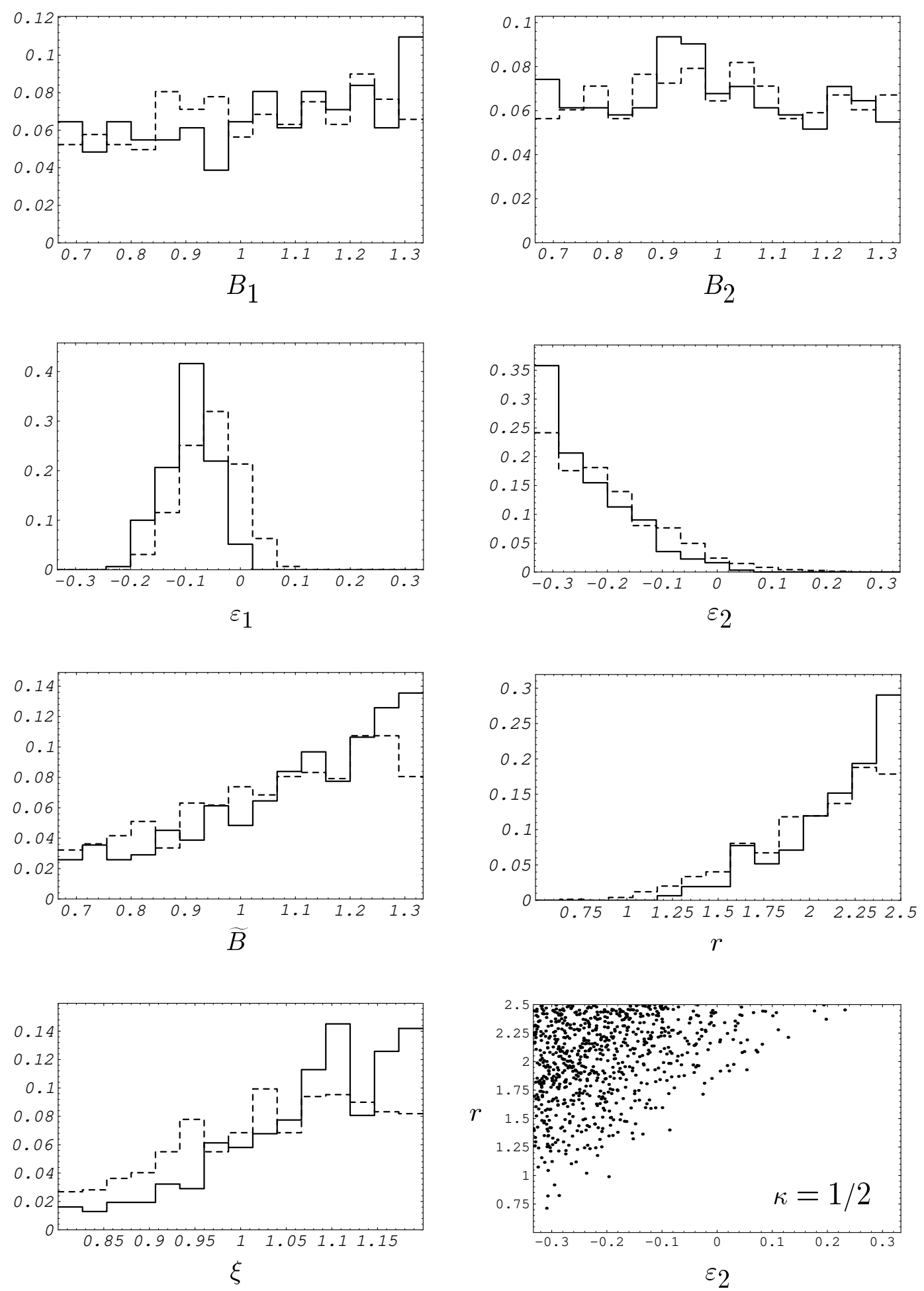

Figure 4: Distributions of the hadronic parameters for the simulations giving results within $2 \sigma$ of the central experimental values. 
to order $1 / m_{b}^{3}$ in the heavy-quark expansion, at which the matrix elements of four-quark operators appear. They describe the physics of nonspectator effects, i.e. contributions in which a light quark in a beauty hadron is actively involved in the weak interaction. We have presented a model-independent study of such contributions introducing a minimal set of hadronic parameters, which eventually may be determined using some field-theoretic approach such as lattice gauge theory. We find that in $B$-meson decays the coefficients of the colour-octet nonfactorizable operators are much larger than those of the colour-singlet factorizable operators, and therefore the contributions from the nonfactorizable operators cannot be neglected. We also find that the theoretical predictions for lifetime ratios still suffer from large perturbative uncertainties, which could only be reduced if the short-distance coefficients in the heavy-quark expansion were calculated to next-to-leading order. The theoretical prediction for the ratio $\tau\left(B^{-}\right) / \tau\left(B^{0}\right)$ is in agreement with experiment; however, our present ignorance about the matrix elements of four-quark operators does not allow us to calculate this ratio with an accuracy of better than about $20 \%$. As a consequence, it is not possible to extract the $B$-meson decay constant $f_{B}$ from a measurement of $\tau\left(B^{-}\right) / \tau\left(B^{0}\right)$.

The short $\Lambda_{b}$ lifetime remains a potential problem for the heavy-quark theory. If the current experimental value persists, either some hadronic matrix elements in the baryon and/or meson sector must be larger than naive expectations, or local quark-hadron duality fails in nonleptonic inclusive decays. In the latter case, the explanation of the puzzle of the $\Lambda_{b}$ lifetime lies beyond our present capabilities. We stress, however, that in view of our results it is premature to conclude a failure of the heavy-quark expansion caused by sizeable duality violations. There is a small region of parameter space where the experimental data can be accommodated by theory.

If indeed the observed pattern of beauty lifetimes is caused by a conspiracy of some large hadronic matrix elements, which are the implications and tests of this scenario? A crucial test will be to prove (or disprove) with a field-theoretical calculation that at least one of the two parameters $\varepsilon_{2}$ and $r$ has the large value required to explain the data. At present, efforts to compute these parameters using lattice gauge theory are under way [29]. An important implication concerns the theoretical understanding of the semileptonic branching ratio and charm yield in $B$ decays. These issues are discussed at length elsewhere in these Proceedings [30]. All theoretical calculations of these quantities so far have neglected nonspectator contributions [31]-33]. The most recent theoretical predictions are [1]

$$
\begin{aligned}
\mathrm{B}_{\mathrm{SL}} & = \begin{cases}12.0 \pm 1.0 \% ; & \mu=m_{b}, \\
10.9 \pm 1.0 \% ; & \mu=m_{b} / 2,\end{cases} \\
n_{c} & = \begin{cases}1.20 \mp 0.06 ; & \mu=m_{b}, \\
1.21 \mp 0.06 ; & \mu=m_{b} / 2,\end{cases}
\end{aligned}
$$

with correlated errors. Notice that the semileptonic branching ratio has a stronger scale dependence than $n_{c}$. By choosing a low renormalization scale, values $\mathrm{B}_{\mathrm{SL}}<11 \%$ can be accommodated. Currently, the experimental values of $B_{\mathrm{SL}}$ and $n_{c}$ are still controversial; however, the value of $n_{c}$ tends to lie below the theoretical predictions. 
Nonspectator contributions can change the predictions for the semileptonic branching ratio significantly; however, they have a negligible effect on $n_{c}$. Irrespective of the precise values of the hadronic parameters, there is a model-independent relation $\Delta n_{c} \approx \Delta B_{\mathrm{SL}} \approx$ $0-1 \%$ [11], i.e. the relative effect on $B_{\mathrm{SL}}$ is ten times larger than that on $n_{c}$. A large negative value of $\varepsilon_{2}$ implies a positive contribution to $B_{\mathrm{SL}}$, which is not preferred by the data. This restriction favours the solution where the $\Lambda_{b}$ lifetime puzzle is solved by a large value of the baryon parameter $r$.

The fact that the charm yield $n_{c}$ is very little affected by nonspectator effects is interesting. To reduce $n_{c}$ significantly below the values quoted in (18) requires an anomalously large branching ratio for charmless nonleptonic $B$ decays. At present, a value of $\mathrm{B}\left(B \rightarrow X_{s}+\right.$ no charm $)$ several times larger than predicted by the Standard Model is not excluded by the data. Such interesting scenarios are explored elsewhere in these Proceedings [34.

\section{Acknowledgements}

Much of the work presented here has been done in a most enjoyable collaboration with Chris Sachrajda, which is gratefully acknowledged. I would also like to thank Tom Browder, Sandip Pakvasa and their staff for making this conference most pleasant and inspiring.

\section{References}

[1] J. Chay, H. Georgi, and B. Grinstein, Phys. Lett. B 247, 399 (1990).

[2] I.I. Bigi, N.G. Uraltsev, and A.I. Vainshtein, Phys. Lett. B 293, 430 (1992) [E: 297, 477 (1993)]; I.I. Bigi, M.A. Shifman, N.G. Uraltsev, and A.I. Vainshtein, Phys. Rev. Lett. 71, 496 (1993); Int. J. Mod. Phys. A 9, 2467 (1994); B. Blok, L. Koyrakh, M.A. Shifman, and A.I. Vainshtein, Phys. Rev. D 49, 3356 (1994) [E: 50, 3572 (1994)].

[3] A.V. Manohar and M.B. Wise, Phys. Rev. D 49, 1310 (1994).

[4] M. Luke and M.J. Savage, Phys. Lett. B 321, 88 (1994); A.F. Falk, M. Luke, and M.J. Savage, Phys. Rev. D 49, 3367 (1994).

[5] A.F. Falk, Z. Ligeti, M. Neubert, and Y. Nir, Phys. Lett. B 326, 145 (1994).

[6] M. Neubert, Phys. Rev. D 49, 3392 and 4623 (1994); T. Mannel and M. Neubert, Phys. Rev. D 50, 2037 (1994).

[7] E.C. Poggio, H.R. Quinn, and S. Weinberg, Phys. Rev. D 13, 1958 (1976). 
[8] M. Shifman, in: Proceedings of the Joint Meeting of the International Symposium on Particles, Strings and Cosmology and the 19th Johns Hopkins Workshop on Current Problems in Particle Theory, Baltimore, Maryland, March 1995, p. 69; B. Chibisov, R.D. Dikeman, M. Shifman, and N. Uraltsev, Int. J. Mod. Phys. A 12, 2075 (1997).

[9] C.G. Boyd, B. Grinstein, and A.V. Manohar, Phys. Rev. D 54, 2081 (1996).

[10] M. Girone and M. Neubert, Phys. Rev. Lett. 76, 3061 (1996).

[11] M. Neubert and C.T. Sachrajda, Nucl. Phys. B 483, 339 (1997).

[12] G. Altarelli and L. Maiani, Phys. Lett. B 52, 351 (1974).

[13] M.K. Gaillard and B.W. Lee, Phys. Rev. Lett. 33, 108 (1974).

[14] For a review, see: M. Neubert, Phys. Rep. 245, 259 (1994); Int. J. Mod. Phys. A 11, 4173 (1996).

[15] A.F. Falk and M. Neubert, Phys. Rev. D 47, 2965 and 2982 (1993).

[16] For a recent discussion, see: M. Neubert, Phys. Lett. B 393, 110 (1997); Preprint CERN-TH/97-19 (1997) hep-ph/9702310, to appear in the Proceedings of the 4th KEK Topical Conference on Flavour Physics, KEK, Japan, October 1996.

[17] Tom Junk, these Proceedings; J. Alcaraz et al. ( $B$ Lifetime Group),

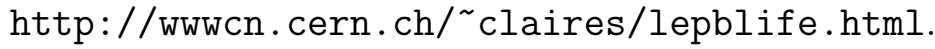

[18] I.I. Bigi and N.G. Uraltsev, Nucl. Phys. B 423, 33 (1994); Z. Phys. C 62, 623 (1994).

[19] M.A. Shifman, A.I. Vainshtein, and V.I. Zakharov, Nucl. Phys. B 147, 385 and 448 (1979).

[20] N. Bilic, B. Guberina, and J. Trampetic, Nucl. Phys. B 248, 261 (1984); B. Guberina, R. Rückl, and J. Trampetic, Z. Phys. C 33, 297 (1986).

[21] M. Shifman and M. Voloshin, Sov. J. Nucl. Phys. 41, 120 (1985); JETP 64, 698 (1986).

[22] B. Guberina, S. Nussinov, R. Peccei, and R. Rückl, Phys. Lett. B 89, 111 (1979).

[23] P. Colangelo and F. De Fazio, Phys. Lett. B 387, 371 (1996).

[24] J.L. Rosner, Phys. Lett. B 379, 267 (1996).

[25] P. Abreu et al. (DELPHI Collaboration), Preprint DELPHI 95-107 PHYS 542 (1995), contributed paper to the International Europhysics Conference on High Energy Physics, Brussels, Belgium, September 1995. 
[26] B. Blok and M. Shifman, in: Proceedings of the 3rd Workshop on the Tau-Charm Factory, Marbella, Spain, June 1993, edited by J. Kirkby and R. Kirkby (Editions Frontieres, 1994).

[27] I.I. Bigi et al., in: B Decays, edited by S. Stone, Second Edition (World Scientific, Singapore, 1994), p. 134; I.I. Bigi, Nuovo Cim. 109A, 713 (1996); Preprint UNDHEP-95-BIG02 (1995) hep-ph/9508408.

[28] P. Abreu et al. (DELPHI Collaboration), Z. Phys. C 74, 19 (1997).

[29] C.T. Sachrajda, private communication.

[30] I. Dunietz, these Proceedings; H. Yamamoto, these Proceedings.

[31] G. Altarelli and S. Petrarca, Phys. Lett. B 261, 303 (1991).

[32] I. Bigi, B. Blok, M.A. Shifman, and A. Vainshtein, Phys. Lett. B 323, 408 (1994).

[33] E. Bagan, P. Ball, V.M. Braun, and P. Gosdzinsky, Nucl. Phys. B 432, 3 (1994); Phys. Lett. B 342, 362 (1995) [E: 374, 363 (1996)]; E. Bagan, P. Ball, B. Fiol, and P. Gosdzinsky, Phys. Lett. B 351, 546 (1995).

[34] A. Kagan, these Proceedings. 